\title{
A numerical model of transformation superplasticity for iron
}

\author{
Peter Zwigl, David C. Dunand* \\ Department of Materials Science and Engineering, Northwestern University, Evanston, IL 60208, USA
}

Received 26 February 1998; received in revised form 7 September 1998

\begin{abstract}
A numerical model of transformation superplasticity for an elastic, ideally plastic material is presented using a two-dimensional plane-strain formulation considering both temperature and displacement. The evolution of temperature, stresses and strains during the $\alpha / \gamma$ phase transformation of iron is computed for different values of the applied stress. For low stresses, the numerical model predicts a linear relationship between the uniaxial applied stress and the uniaxial plastic strain increment accumulated after crossing the phase transformation range. For high stresses, the relationship becomes non-linear: the strain increments tend to infinity as the applied stress approaches the yield stress. Both of these trends are in qualitative agreement with existing analytical solutions for transformation superplasticity in an ideally plastic material. Furthermore, upon introducing plane-strain specific equivalent quantities for the transformation mismatch and the yield stress, the numerical model is in good quantitative agreement with both analytical predictions and experimental data for pure iron. (C) 1999 Elsevier Science S.A. All rights reserved.
\end{abstract}

Keywords: Superplasticity; Iron; Transformation; Finite-element model

\section{Introduction}

Transformation superplasticity is a deformation mechanism relying on the biasing by an externally applied stress of isotropic internal mismatch stresses or strains induced during the phase transformation of allotropic polycrystalline materials. Plastic deformation of the weaker phase due to the internal and external stresses determines the macroscopic strain increment developed during the allotropic transformation. As internal stresses are regenerated each time the material transforms, large superplastic strains can result by accumulation of the individual strain increments produced during cycling about the allotropic temperature [1-4].

For elastic, ideally-plastic materials, Greenwood and Johnson [5] derived an approximate analytical solution for the uniaxial strain increment $\Delta \varepsilon$ accumulated during a full temperature cycle (where the allotropic temperature is crossed twice), as a function of the absolute value of the volume mismatch between the two al-

\footnotetext{
Corresponding author. Tel.: + 1-847-491-5370; fax: + 1-847-4676573. $\alpha \sim \frac{5}{6} \delta$

lotropic phases $\Delta V / V$, the externally applied uniaxial stress $\sigma$ and the uniaxial yield stress $\sigma_{\mathrm{Y}}$ of the weaker phase:

$\Delta \varepsilon \sim \frac{5}{3} \frac{\Delta V}{V} \frac{\sigma}{\sigma_{\mathrm{Y}}}$

However, Eq. (1) is valid only for applied stresses which are small compared to the yield stress [6]. Based on Greenwood and Johnson's derivation, [6,7] the model can be extended for all applied stresses below the yield stress:

$$
\begin{gathered}
\delta=\frac{1}{4}+\frac{1}{6 \alpha}+\frac{1}{2 \sqrt{2 \alpha}}\left(\frac{3 \alpha}{4}-\frac{1}{6}-\frac{1}{9 \alpha}\right) \\
\ln \left[\frac{(3 \alpha+3 \sqrt{2 \alpha}+2)^{2}}{9 \alpha^{2}-6 \alpha+4}\right]
\end{gathered}
$$

where the dimensionless parameters $\delta=\sigma / \sigma_{\mathrm{Y}}$ and $\alpha=$ $(\Delta \varepsilon / 2) /(\Delta V / V)$ have been introduced. Eq. (2) was found to be in good agreement with experimental data for pure iron undergoing the $\alpha / \gamma$ transformation without strain-hardening [6]. As expected, Eq. (2) tends toward the limit for small stresses (Eq. (1)) expressed in a dimensionless manner for half a temperature cycle as: 
While the closed-form solution Eq. (2) is compact and predictive, it does not give any information about the time-evolution of internal or macroscopic strains during the allotropic transformation. Such information can however be generated by numerical models, which can also take into account the temperature-dependence of the thermo-mechanical properties during thermal cycling and the coupling between thermal and mechanical behavior of the material. Numerical modeling has been used to study the related problems of thermal mismatch superplasticity in $\mathrm{Al}-\mathrm{SiC}$ composites during thermal cycling [8,9], and transformation plasticity in steel by formation of pearlite [10] or martensite [11-14]. The origin of the internal stress in these models is based on: (1) full-bonding at the phase-boundaries for composites; (2) unit-cell constraints; and (3) reactionstresses caused by a two-dimensional representation of a three-dimensional state of stress (e.g. plane stress assumes a stress-free surface and plane strain a reaction stress along the 3-direction). Despite these simplifications, the results of these numerical models are in agreement with experimental observations and analytical predictions and thus validate the above assumptions.

In the present paper, we develop a numerical model for a material undergoing an allotropic phase transformation under an externally applied stress. The validity of the numerical model is established through comparison of the numerical results for the case of iron to predictions of the analytical model given by Eq. (2) and experimental results [5,15]. Furthermore, the consistency of the numerical model with respect to changes of the yield stress and of the allotropic volume mismatch is tested and the evolution of strains during the cycle is discussed.

\section{Model}

Based on the successful numerical description presented in the literature for thermal mismatch superplasticity in composites $[8,9]$ and transformation-induced plasticity [11-14] (i.e. deformation by shear), a two-dimensional representation is sufficient to capture the physical behavior of the complex three-dimensional problem of transformation plasticity modeled by Eqs. (2) and (3). In the present approach, the mismatch is not generated locally at the boundary between the two allotropic phases; rather, the mismatch occurs uniformly over the whole domain as a result of the planestrain constraint. A plane-strain formulation is preferable to the use of an axial-symmetric state of stress, which introduces a radial dimension as an additional arbitrary quantity. Also, the axial-symmetric state of stress approaches plane-strain conditions at large radii. Finally, a plane-stress state is not suitable as no mismatch would be produced in the domain. Other two-dimensional stress states such as generalized planestrain could be used, but the potential gains are marginal given the additional complexity.

The current work explores the simple base-line case of a transforming, ideally-plastic material showing no strain-hardening, for which both analytical solutions and experimental data exist. Once the validity of the simple model is established, it could be extended with some confidence to the more complex cases of strainhardening, time-dependent deformation and multiphase materials.

The numerical model consists of 16 plane-strain, temperature-displacement elements [16] regularly arranged in a square $4 \times 4$ mesh with a length $L_{0}=100$ $\mu \mathrm{m}$ as shown in Fig. 1. The boundary- and symmetry conditions are defined such that the mesh remains rectangular throughout the analysis. The initial temperature of the stress-free domain was set to $T=909^{\circ} \mathrm{C}$. Defining the 3-direction as being constrained by planestrain (i.e. $\varepsilon_{33}=0$ ), an external uniaxial stress $\sigma_{22}$ (referred to as $\sigma$ in the following) is applied along the a-a edge of the mesh for the first step of the analysis. In the following steps, a square temperature profile (Fig. 1) is repeatedly applied along the $\mathrm{b}-\mathrm{b}$ edge of the mesh where the temperature is cycled between $T_{1}=909^{\circ} \mathrm{C}$ and $T_{\mathrm{u}}=915^{\circ} \mathrm{C}$ about the $\alpha-\gamma$ allotropic range of iron, taken as $911.5-912.5^{\circ} \mathrm{C}$. The temperature isotherms are perpendicular to the direction of the applied stress so that ratchetting is possible. The time-incrementation during the transient temperature-displacement analysis (performed with ABAQUS version 5.5 [16]) is set so that the element strain is below $5 \times 10^{-4}$ and the temperature difference is below $0.2 \mathrm{~K}$ during each increment. Heat transport is assumed to occur by convection with a heat transfer coefficient $h=50 \mathrm{~W} \mathrm{~m}^{-2} \mathrm{~K}^{-1}$. Consis-

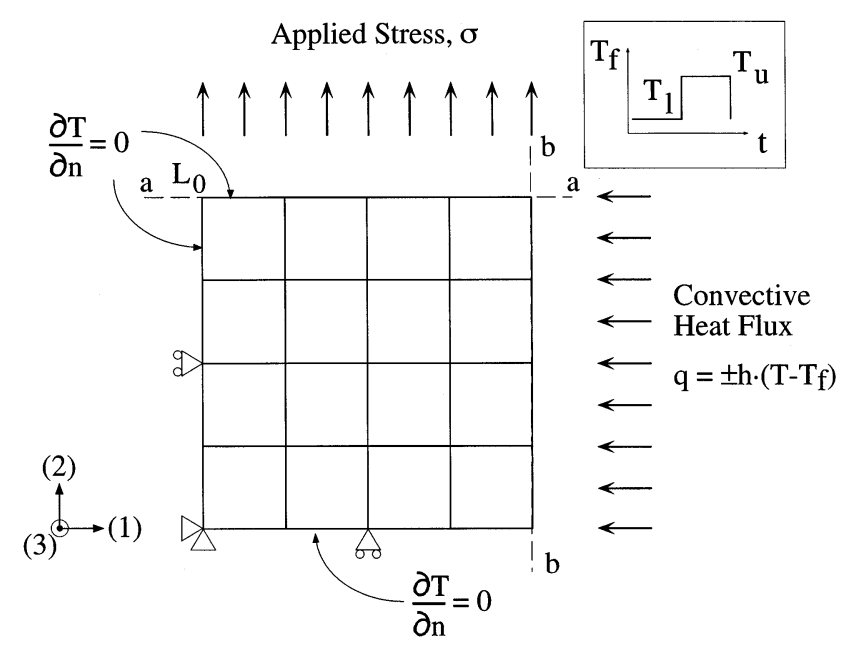

Fig. 1. Mesh, symmetry and boundary conditions of the plane-strain, temperature-displacement model. The applied temperature profile is given in insert. 
Table 1

Physical properties of iron near the phase transformation

\begin{tabular}{|c|c|c|c|c|}
\hline \multirow[t]{2}{*}{ Property } & & \multicolumn{2}{|l|}{ Temperature } & \multirow[t]{2}{*}{ Reference } \\
\hline & & $911.5^{\circ} \mathrm{C}(\alpha$-iron $)$ & $912.5^{\circ} \mathrm{C}(\gamma$-iron $)$ & \\
\hline Density & $\rho\left(\mathrm{g} \mathrm{cm}^{-3}\right)$ & 7.87 & 7.58 & {$[18]$} \\
\hline Transformation enthalpy & $\Delta H\left(\mathrm{~J} \mathrm{~g}^{-1}\right)$ & 18.8 & & {$[19]$} \\
\hline Specific heat & $C_{\mathrm{P}}\left(\mathrm{J} \mathrm{g}^{-1} \mathrm{~K}^{-1}\right)$ & 0.87 & 0.71 & {$[19]$} \\
\hline Coefficient of thermal expansion ${ }^{a}$ & $\alpha_{20^{\circ} \mathrm{C}}\left(\mathrm{K}^{-1}\right) \cdot 10^{6}$ & 15.0 & 11.1 & {$[17]$} \\
\hline Thermal conductivity & $\mathrm{k}\left(\mathrm{W} \mathrm{cm}-1 \mathrm{~K}^{-1}\right)$ & 0.296 & 0.286 & {$[20]$} \\
\hline Poisson's ratio & $v(-)$ & $0.3^{\mathrm{b}}$ & 0.3 & {$[18]$} \\
\hline Young's modulus & $E(\mathrm{GPa})$ & 65.1 & 117 & {$[21]$} \\
\hline
\end{tabular}

${ }^{a}$ Technical value, defined as $\alpha_{20^{\circ} \mathrm{C}}=\left(L-L_{20}{ }^{\circ} \mathrm{C}\right) /\left(T-20^{\circ} \mathrm{C}\right)$ where $L$ and $L_{20^{\circ} \mathrm{C}}$ are the sample lengths at temperatures $T\left({ }^{\circ} \mathrm{C}\right)$ and $20^{\circ} \mathrm{C}$, respectively. This value for $\gamma$-iron incorporates the allotropic contraction.

${ }^{\mathrm{b}} v=0.5$ was used during yield.

tent with the analytical model, isotropic macroscopic material properties are used to model the $\alpha-\gamma$ transformation of iron, as summarized in Table 1 .

As shown in Fig. 2 for the thermal strain, the density change is modeled by varying the technical coefficient of thermal expansion $\alpha_{20^{\circ} \mathrm{C}}$ linearly over the allotropic temperature range between the values given in Table 1 . The technical coefficient of thermal expansion includes the contraction due to the $\alpha / \gamma$-iron transformation with respect to room-temperature and thus has a smaller value for $\gamma$-iron than for $\alpha$-iron. However, the instantaneous thermal expansion, defined as the slope of the extension/temperature curve, is higher for $\gamma$-iron than for $\alpha$-iron. Using technical coefficients of thermal expansion, the allotropic length change is:

$\frac{\Delta L}{L}=\left(\alpha_{20^{\circ} \mathrm{C}, \alpha}-\alpha_{20^{\circ} \mathrm{C}, \gamma}\right) \cdot \Delta T$

With $\alpha_{20^{\circ} \mathrm{C}, \alpha}$ and $\alpha_{20^{\circ} \mathrm{C}, \gamma}$ taken from Table 1, and with $\Delta T=912-20^{\circ} \mathrm{C}$, Eq. (4) gives $\Delta L / L=0.348 \%$, corresponding to a volume change $(\Delta V / V)=3 \cdot(\Delta L / L)=$ $1.04 \%$, in agreement with the value reported for iron [17].

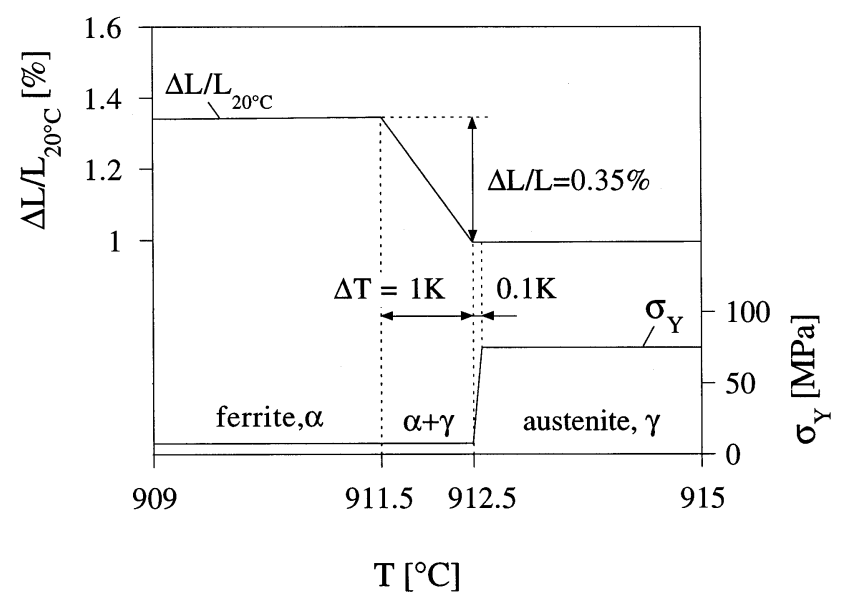

Fig. 2. Thermal strain $\left(\Delta L / L_{20^{\circ} \mathrm{C}}\right)$ with allotropic strain $(\Delta L / L)$ and yield stress $\sigma_{\mathrm{Y}}$ as a function of temperature.
The yield stress of the low-temperature $\alpha$-iron is taken as $\sigma_{\mathrm{Y}}=7.5 \mathrm{MPa}$, as determined from experimental transformation superplasticity data [6]. It is assumed that the yield stress of the material is equal to that of $\alpha$-iron throughout the transformation (i.e. over $\Delta T=1$ $\mathrm{K}$ ), and that it increases rapidly to that of $\gamma$-iron immediately after the end of the phase transformation over a small temperature interval $\Delta T=0.1 \mathrm{~K}$, as shown in Fig. 2. Since $\gamma$-iron is much stronger than $\alpha$-iron, we assume a factor ten between the yield stresses of the two phases, but our results are insensitive to the exact value of this factor, as long as $\gamma$-iron remains elastic throughout deformation.

\section{Results}

Fig. 3(a) shows the time-dependence of the strain $\varepsilon_{22}$ (referred to as $\varepsilon$ in the following) and the domain temperature during the first three thermal cycles for an applied stress $\sigma=4.0 \mathrm{MPa}$. The cycle period is $\Delta t=240$ $\mathrm{s}$, with equal intervals $\Delta t_{1 / 2}=120 \mathrm{~s}$ for heating and cooling. The temperature was determined at the upper right hand corner of the domain and thermal gradients within the material were below $\Delta T=0.1 \mathrm{~K}$ at all times. During the heating stage of the cycles, the temperature increases rapidly from $T=909^{\circ} \mathrm{C}$ to the onset of the phase transformation, modeled at $T=911.5^{\circ} \mathrm{C}$. Because of the heat absorbed or released during the phase transformation, the heating and cooling rates are reduced when the material transforms (Fig. 3(a)).

A small thermal expansion is observed upon initial heating (Fig. 3(b)) before the domain shrinks over the phase transformation interval $\Delta T=1 \mathrm{~K}$. This large allotropic contraction is followed by a small thermal expansion (Fig. 3(c)) upon heating to the upper cycling temperature $T=915^{\circ} \mathrm{C}$. On cooling, thermal contractions occur outside the phase transformation interval over which the material expands (Fig. 3(c,d)). An overall plastic strain increment in the 2-direction (referred 

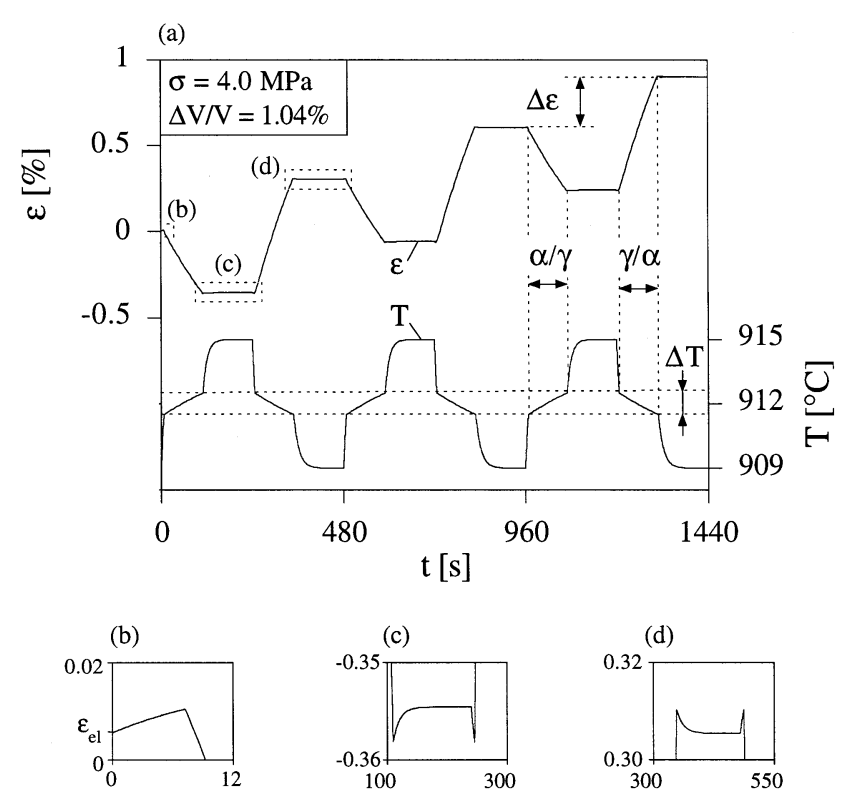

Fig. 3. (a) Total uniaxial strain in the 2-direction and temperature as a function of time during the first three cycles for an applied stress $\sigma=4.0 \mathrm{MPa}$ and a uniaxial yield stress at $\sigma_{\mathrm{Y}}=7.5 \mathrm{MPa}$; (b,c,d) are magnified regions of the strain history.

to as $\Delta \varepsilon$ in the following) is observed after each full cycle. The magnitude of the strain increment, which was taken as the difference of plastic strains between the start and the end of a cycle, changes slightly between the first and the succeeding cycles, because of different residual stresses: at the onset of the first heating ramp, the material is free of residual stresses, but at the onset of the second and all subsequent heating ramps, residual stresses exist in the material. The value of $\Delta \varepsilon$ remains unchanged after the first cycle.

Fig. 4 shows the steady-state strain increment in the 2-direction (i.e. $\Delta \varepsilon$ for the third temperature cycle) as a

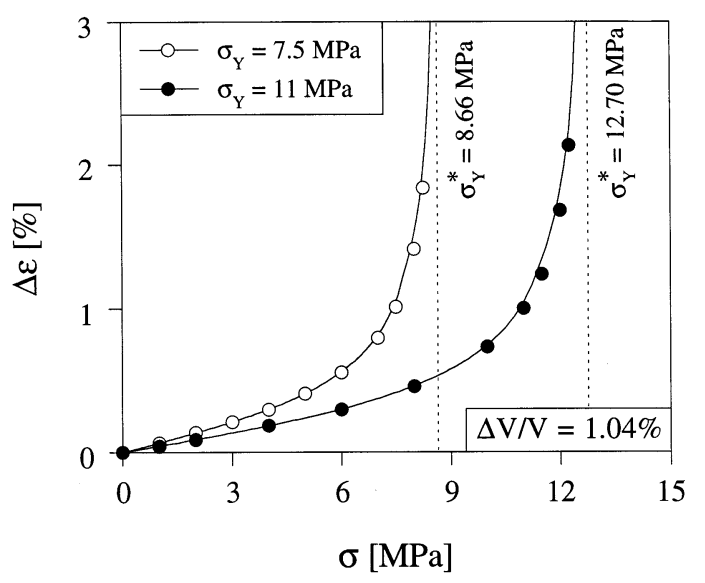

Fig. 4. Strain increment in the 2-direction for a full cycle as a function of the applied stress for uniaxial yield stresses $\sigma_{\mathrm{Y}}=7.5$ and $11 \mathrm{MPa}$, respectively.

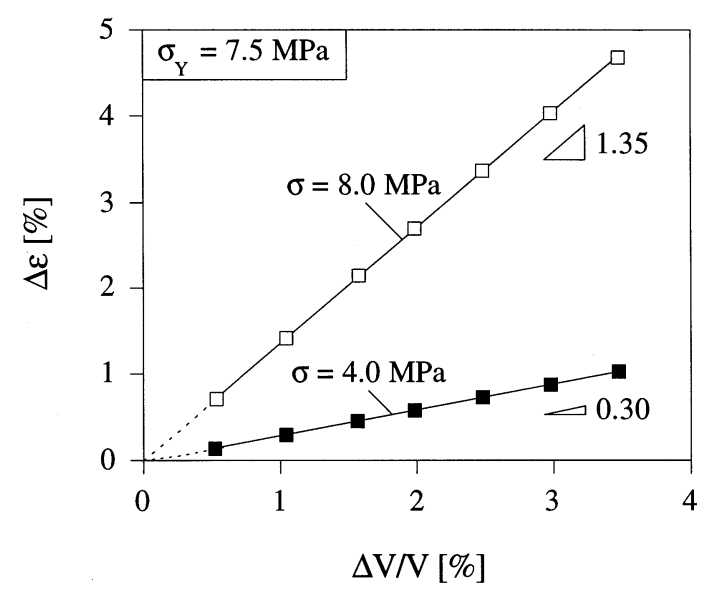

Fig. 5. Strain increment in the 2-direction for a full cycle as a function of the allotropic volume mismatch for applied stresses $\sigma=4.0$ and 8.0 MPa respectively with a uniaxial yield stress $\sigma_{\mathrm{Y}}=7.5 \mathrm{MPa}$.

function of the applied stress. A linear relationship between the strain increment and the applied stress exists at low stresses, while progressively increasing strain increments are developed at high stress levels. Also, finite strain increments are obtained above the uniaxial yield stress $\sigma_{\mathrm{Y}}$ because of the plane-strain condition, as discussed later. When higher values are taken for the yield stress limits $\left(\sigma_{\mathrm{Y}}=11 \mathrm{MPa}\right.$ for $\alpha$-iron and $\sigma_{\mathrm{Y}}=110 \mathrm{MPa}$ for $\gamma$-iron), smaller strain increments are observed at low stresses and the divergence occurs at a higher applied stress.

To examine the sensitivity of the model to the value of the allotropic volume mismatch $\Delta V / V$, the technical coefficient of thermal expansion of $\gamma$-iron was further varied, i.e. $\alpha_{20{ }^{\circ} \mathrm{C}, \gamma}=\alpha_{20^{\circ} \mathrm{C}, \alpha}-(\Delta V / V) /(3 \cdot \Delta T)$. Fig. 5 shows the effect of the volume mismatch on the strain increment per cycle for applied stresses of $\sigma=4.0$ and 8.0 $\mathrm{MPa}$, respectively, using a uniaxial yield stress of $\sigma_{\mathrm{Y}}=7.5 \mathrm{MPa}$. The strain increment is proportional to the volume mismatch, with slopes $d(\Delta \varepsilon) / d(\Delta V / V)=$ 0.30 and 1.35 at the lower and higher applied stress, respectively.

\section{Discussion}

\subsection{Temperature-, strain-and stress-evolution}

The numerical results showed that thermal gradients were insignificant $(\Delta T<0.1 \mathrm{~K})$ with the standard 16-element mesh (or with meshes containing 64 and 256 elements). This is consistent with the value much smaller than unity for the Biot number:

$\mathrm{Bi}=\frac{h \cdot L_{0}}{k}$ 
where $k$ is the thermal conductivity and $L_{0}$ is the domain length (Fig. 1), with values $h=0.005 \mathrm{~W}$ $\mathrm{cm}^{-2} \cdot \mathrm{K}^{-1}, k=0.3 \mathrm{~W} \mathrm{~cm}^{-1} \mathrm{~K}^{-1}, \mathrm{~L}_{0}=0.01 \mathrm{~cm}$, Eq. (5) gives $\mathrm{Bi}=1.7 \cdot 10^{-4}$. Under these thermal conditions, the time for transformation is:

$\Delta t=\frac{L_{0} \cdot \rho \cdot \Delta H}{h \cdot\left|T_{\mathrm{S}}-T_{\mathrm{P}}\right|}$

where $\rho$ is the density, $\Delta H$ is the transformation enthalpy, $T_{\mathrm{S}}$ is the surface temperature $\left(T_{\mathrm{S}}=915^{\circ} \mathrm{C}\right.$ and $909^{\circ} \mathrm{C}$ on heating and cooling, respectively) and $T_{\mathrm{P}}$ is the phase transformation temperature. Since the phase transformation is modeled over a temperature interval $\Delta T=1 \mathrm{~K}$, Eq. (6) is integrated between $T_{\mathrm{P} 1}=911.5^{\circ} \mathrm{C}$ (or $912.5^{\circ} \mathrm{C}$ ) and $T_{\mathrm{P} 2}=912.5^{\circ} \mathrm{C}$ (or $911.5^{\circ} \mathrm{C}$ ) for heating (or cooling):

$\Delta t=\frac{L_{0} \cdot \rho \cdot \Delta H}{h \cdot\left|T_{\mathrm{P} 2}-T_{\mathrm{P} 1}\right|} \cdot \ln \left(\frac{T_{\mathrm{P} 1}-T_{\mathrm{S}}}{T_{\mathrm{P} 2}-T_{\mathrm{S}}}\right)$

Taking an average density of $\rho=7.73 \mathrm{~g} \mathrm{~cm}^{-3}$, the expected time for transformation from Eq. (7) is $\Delta t=$ $98 \mathrm{~s}$, close to the value found numerically $(\Delta t=102 \mathrm{~s}$ for Fig. 3(a)).

Based on the elastic responses $\varepsilon_{\mathrm{el}}$ of the domain upon initial application of a series of externally applied uniaxial stresses (e.g. Fig. 3(b) for $\sigma=4.0 \mathrm{MPa}$ ), the numerical elastic modulus for $\alpha$-iron at $T=909^{\circ} \mathrm{C}$ is $E^{*}=71.6 \mathrm{GPa}$, in good agreement with the expected value of the elastic modulus in plane-strain $E^{*}=E$ / $\left(1-v^{2}\right)=71.5 \mathrm{GPa}$ (where $v$ is Poisson's ratio), using elastic constants given in Table 1.

The magnitudes of the thermal strains outside the phase transformation range (Fig. 3(c,d)) are small compared to transformation strains (Fig. 3(a)), so that thermal expansion mismatch plasticity can be excluded as a deformation mechanism. Furthermore, because the thermal and allotropic strains have opposite signs, the small thermal mismatch outside the transformation range reduces the allotropic mismatch produced during transformation.

As shown in Fig. 3(a) for an applied stress $\sigma=4.0$ $\mathrm{MPa}$, the sample shrinks in the 2-direction during heating through the transformation by $\Delta \varepsilon_{\mathrm{h}}=-0.370 \%$ and expands on cooling by $\Delta \varepsilon_{\mathrm{c}}=0.669 \%$, giving a strain increment over a whole cycle of $\Delta \varepsilon=\Delta \varepsilon_{\mathrm{h}}+\Delta \varepsilon_{\mathrm{c}}=$ $0.299 \%$. The magnitude of $\Delta \varepsilon_{\mathrm{h}}$ and $\Delta \varepsilon_{\mathrm{c}}$ are different because on cooling the allotropic strain $\Delta L / L$ has the same sign as the plastic strain due to the applied stress, while on heating the strains have opposite signs. When no stress is applied, the uniaxial allotropic strains developed on heating and cooling in the 2-direction are equal and of opposite sign: $\left|\varepsilon_{0}\right|=0.516 \%$. This strain translates into a volume change $\Delta V / V=2 \varepsilon_{0}=1.032 \%$ for plane-strain conditions, close to the allotropic volume mismatch $(\Delta V / V=1.048 \%)$.

\subsection{Model discussion}

The model is sensitive to the assumption made for the temperature-dependence of the yield stress during the transformation. The present results are for the specific case where plasticity occurs throughout the transformation range at the lowest yield stress of $\alpha$-iron (Fig. 2). Physically, this is equivalent to plastic deformation being spatially localized in the weak $\alpha$-iron until that phase disappears. The continuum approach taken in the numerical model approximates this spatially inhomogeneous behavior by assuming that the whole domain yields at the yield stress of the $\alpha$-iron. If instead a rule of mixture is used for the yield stress, much reduced plasticity is found.

The nature of the mismatch needs to be examined, since the numerical model allows for two possible types of mismatch. Firstly, internal mismatch stresses are produced by the constraining effect in the 3-direction from the plane-strain condition, i.e. transformation mismatch plasticity and thermal mismatch plasticity. Secondly, since in the present case the material is heated and cooled from one side (Fig. 1), a phase front may form and move in the 1-direction through the material and mismatch stresses can then arise locally near the phase front, i.e. ratchetting. However, no strain increment was observed after a full temperature cycle under zero applied stress (Fig. 4), indicating that ratchetting was absent. This is further confirmed by the lack of thermal gradient discussed earlier.

Because the temperature-, stress- and strain-fields are spatially almost completely homogeneous, the domain presented in Fig. 1 could be replaced by a single element. While this would reduce the computational effort, the non-linearities introduced by the material properties prevent a further reduction to an analytical formulation. The numerical results were insensitive to changes of the cell geometry and domain length. Very small domains or very large heat transfer coefficients could however induce ratchetting.

The numerical results shown in Fig. 4 are in qualitative agreement with the prediction of the analytical solution (Eq. (2)): a linear strain-stress behavior at low stresses is followed by a non-linear strain increase at high stresses. Thus, the numerical model shows the fundamental physical behavior of phase-transformation superplasticity, as observed experimentally and predicted analytically. Besides the possibility of qualitative comparisons to other materials (e.g. to compare transformation superplasticity of iron with that of cobalt), the model can be used for parametric studies, such as the effect of the volume mismatch on the strain increment (Fig. 5). In what follows, an attempt is made to incorporate model-specific assumptions which allow a direct quantitative comparison to analytical models and experimental results. 
The fundamental difference between the analytical and numerical models is the origin of the allotropic mismatch. In the three-dimensional analytical model leading to Eq. (2), internal stresses occur because the transformation is constrained within a plastically deforming material. In the two-dimensional numerical plane-strain model however, the constraint along the 3 -direction is the source of the internal stresses. Thus, the internal stress field is caused by the constraint in one single dimension (the 3-direction), as compared to the constraint in all three dimensions in the analytical model. Thus, to a first approximation, the mismatch in the numerical two-dimensional model is lower by a factor three than the mismatch in the analytical threedimensional model. Then, to compare these models, an equivalent mismatch in plane-strain $(\Delta V / V)^{*}$ is defined as:

$\left(\frac{\Delta V}{V}\right)^{*}=\frac{1}{3}\left(\frac{\Delta V}{V}\right)$

In the present case, $(\Delta V / V)^{*}=(\Delta L / L)=0.348 \%$.

Another important difference between the models is the stress at which plasticity occurs. While the analytical model is based on yield under uniaxial tension, the current numerical model considers yield under planestrain conditions. For plane-strain under an applied stress $\sigma$ in the 2-direction, the non-zero stress components are: $\sigma_{22}=\sigma$, and $\sigma_{33}=v \sigma$. Thus, the equivalent Von Mises stress is:

$\sigma_{\text {eq }}=\sigma \cdot \sqrt{1-v+v^{2}}$

and the uniaxial stress in the 3-direction to induce plastic yielding $\sigma_{\mathrm{Y}}^{*}$ is:

$\sigma_{\mathrm{Y}}^{*}=\frac{\sigma_{\mathrm{Y}}}{\sqrt{1-v+v^{2}}}$.

Taking $v=1 / 2$, Eq. (10) gives $\sigma_{\mathrm{Y}}^{*}=(2 / \sqrt{3}) \sigma_{\mathrm{Y}}$.

For the specific case of Fig. 4 with a uniaxial yield stress $\sigma_{\mathrm{Y}}=7.5 \mathrm{MPa}$, the plane-strain yield stress calculated from Eq. (10) is then $\sigma_{\mathrm{Y}}^{*}=8.66 \mathrm{MPa}$. As expected, the strain increments in Fig. 4 are finite above $\sigma_{\mathrm{Y}}$ but tend to infinity when approaching $\sigma_{\mathrm{Y}}^{*}$. The same overall behavior is observed in Fig. 4 for the higher yield stress $\sigma_{\mathrm{Y}}=11 \mathrm{MPa}$. At small stresses, the strain increments are proportional to the applied stress, but the proportionality constant is smaller than for $\sigma_{\mathrm{Y}}=$ $7.5 \mathrm{MPa}$, as expected from the larger value of $\sigma_{\mathrm{Y}}$ and Eq. (1). At high stresses, the curve diverges when the stress approaches the plane-strain yield stress $\sigma_{\mathrm{Y}}^{*}=$ $12.70 \mathrm{MPa}$.

The plane-strain specific volume mismatch (Eq. (8)) and yield stress (Eq. (10)) can be used to renormalize the analytical solutions using

$\alpha^{*}=\frac{\Delta \varepsilon}{2 \cdot(\Delta V / V)^{*}}$
$\delta^{*}=\frac{\sigma}{\sigma_{\mathrm{Y}}^{*}}$

instead of $\alpha$ and $\delta$ in Eqs. (2) and (3). The numerical results (Fig. 5) confirm the linearity between the mismatch $\Delta V / V$ and the strain increment in the 2-direction $\Delta \varepsilon$. The results at the lower stress $\sigma=4.0 \mathrm{MPa}$ can be compared to Eq. (3) using the specific plane-strain quantities (Equation 11(a,b)). With $\delta^{*}=0.46$, Eq. (3) predicts $\alpha^{*}=0.38$. This is to be compared to the slope of $d(\Delta \varepsilon) / d(\Delta V / V)=0.30$ in Fig. 5, which must be multiplied by a factor $3 / 2$ to account for a half cycle (factor 2, Equation 11(a)) and for the effective mismatch (factor 3, Eq. (8)), resulting in a value of 0.45 . For a high stress $\sigma=8.0 \mathrm{MPa}\left(\delta^{*}=0.92\right)$ where the strain increments increase non linearly with the applied stress, Eq. (2) gives $\alpha^{*}=3.0$, while $(3 / 2) \cdot d(\Delta \varepsilon) / d(\Delta V /$ $V)=2.0$ is obtained from Fig. 5. The numerical model thus underestimates the analytical normalized strain increment, probably as a result of the different assumptions used.

Fig. 6 shows the numerical values of Fig. 4 normalized by the plane-strain mismatch $(\Delta V / V)^{*}$ and the plane-strain yield stress $\sigma_{\mathrm{Y}}^{*}$ given by Eqs. (8) and (10). Also shown in Fig. 6 are the predictions from Eqs. (2) and (3) (using $2 \cdot \alpha$ for a full temperature cycle encompassing both phase transformations) as well as experimental data on pure iron $[5,15]$. In the linear region at low stresses $(\delta<0.4)$, there is good agreement between the numerical, analytical and experimental results. In

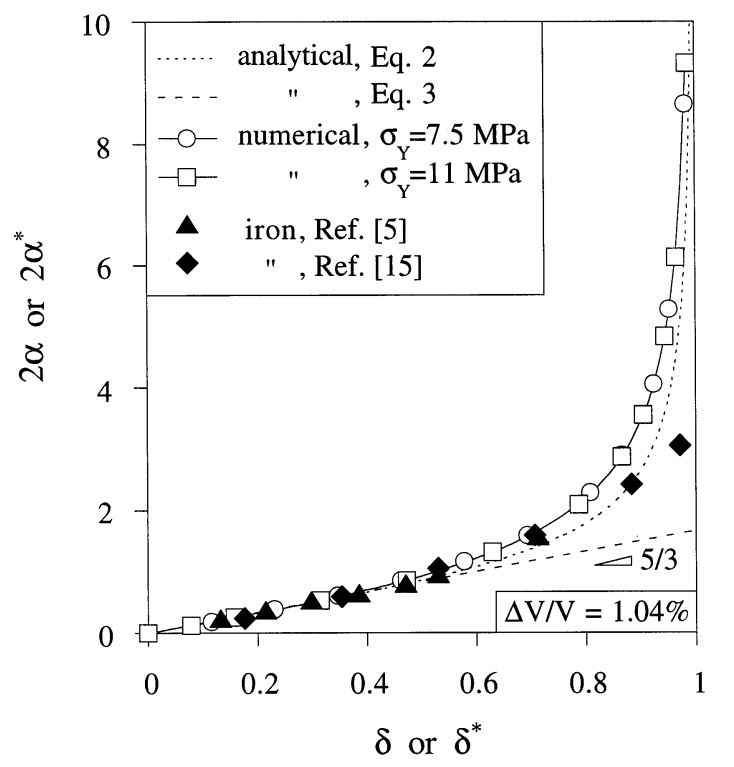

Fig. 6. Strain increment in the 2-direction for a full cycle normalized by the transformation mismatch as a function of the applied stress normalized by the yield stress. Comparison between analytical predictions, numerical predictions and experimental results for pure iron. 
the non-linear region however, the two-dimensional numerical model gives somewhat higher strain values than the three-dimensional analytical solution (Eq. (2)), but correctly predicts the progressive departure from the linear behavior observed both in the analytical model and the experimental data. Also, the experimental point for the highest stress is significantly lower than predicted by the models, probably because the large strains developed during cycling give raise to strain hardening, thus increasing the yield stress of the material and decreasing the total strain increment, as modeled in [6].

\section{Conclusions}

A numerical model for transformation superplasticity is presented for an elastic, ideally-plastic material, where the thermally-induced transformation generates the internal stresses. Numerical results for the $\alpha / \gamma$-iron phase transformation qualitatively capture the behavior predicted by existing closed-form solutions, i.e. the uniaxial strain increments after a thermal cycle increase first linearly with the uniaxial applied stress, but diverge when the applied stress becomes large. However, the numerical model approximates the constrained phase transformation in a polycrystalline material with a simple average plane-strain constraint. Thus, while the constrained mismatch for a polycrystalline material is $\Delta V / V$, only one third of this volumetric mismatch is available as the mismatch source in the plane-strain configuration, where only one direction is constrained. Furthermore, the yield stress in plane-strain is slightly higher than the yield stress under uniaxial conditions. Taking these two differences into account in the normalization of the data, the numerical model can be quantitatively compared to the analytical model as well as experimental data for iron. Good agreement is then found both for small stresses (where strain increments increase linearly with stress) and for high stresses (where the behavior is non-linear).

\section{Acknowledgements}

This study was supported by the US Army Research Office under grant DAAH004-95-1-0629 monitored by Dr W.C. Simmons. The authors also thank Professor H. Lehar (Innsbruck University) for useful discussions and the computer center of Innsbruck University on which workstations all calculations were performed.

\section{References}

[1] J.W. Edington, K.N. Melton, C.P. Cutler, Progress Mater. Sci. 21 (1976) 61.

[2] K.A. Padmanabhan, G.J. Davies, Superplasticity, Springer, London, 1980.

[3] T.J. Nieh, J. Wadsworth, O.D. Sherby, Superplasticity in Metals and Ceramics, Cambridge University Press, Cambridge, 1997.

[4] D.C. Dunand, in: T. Chandra, T. Sakai (Eds.), Thermec'97, TMS, 1997, p. 1821.

[5] G.W. Greenwood, R.H. Johnson, Proc. R. Soc. Lond. 283 (1965) 403.

[6] P. Zwigl, D.C. Dunand, Acta Mater. 45 (1997) 5285.

[7] F.D. Fischer, Acta Metall. Mater. 38 (1990) 1535.

[8] H. Zhang, G.S. Daehn, R.H. Wagoner, Scripta Metall. Mater. 24 (1990) 2151.

[9] H. Zhang, G.S. Daehn, R.H. Wagoner, Scripta Metall. Mater. 25 (1991) 2285

[10] J.F. Ganghoffer, S. Denis, E. Gautier, A. Simon, S. Sjöström, Eur. J. Mech. A/Solids 12 (1993) 21.

[11] S. Denis, S. Sjöström, A. Simon, Met. Trans. 18A (1987) 1203.

[12] E. Gautier, A. Simon, B. Beck, Acta Metall. 35 (1987) 1367.

[13] V.I. Levitas, E. Stein and A.V. Idesman, J. Phys. IV, 6 (1996) C1 309.

[14] F.G. Rammerstorfer, F.D. Fischer, H.J. Böhm, W. Daves, Comput. Struct. 44 (1992) 453.

[15] F.W. Clinard, O.D. Sherby, Acta Metall. 12 (1964) 911.

[16] Hibbet, Karlsson, Sorensen, ABAQUS (Version 5.5), Providence RI.

[17] Z.S. Basinski, W. Hume-Rothery, A.L. Sutton, Proc. R. Soc. Lond. 229 (1955) 459.

[18] Metals Handbook: Properties and Selection: Nonferrous Alloys and Pure Metals, Am. Soc. Met., Metals Park, OH, 1979.

[19] I. Barin, Thermochemical Properties of Inorganic Substances, Purdue University Press, Purdue, 1975.

[20] Y.S. Touloukian, Thermophysical Properties of High Temperature Materials, Plenum, New York, vol. 1, 1970.

[21] H.J. Frost, M.F. Ashby, Deformation-Mechanism Maps: The Plasticity and Creep of Metals and Ceramics, Pergamon, Oxford, 1982. 